\title{
DESENVOLVIMENTO DE UM DISPOSITIVO DE ADMINISTRAÇÃO DE INSULINA PARA CRIANÇAS DIABÉTICAS
}

\author{
Lucas Renato Tescke (MSc) \\ Instituto Federal Farroupilha Câmpus Santa Rosa \\ lucas.tescke@iffarroupilha.edu.br
}

João Manuel R. S. Tavares (PhD)

Instituto de Engenharia Mecânica e Gestão Industrial

Faculdade de Engenharia da Universidade do Porto

tavares@fe.up.pt

Isa C. T. Santos (MSc, PhD)
isa.santos@fe.up.pt

\begin{abstract}
Resumo: Este projeto visou o desenvolvimento de um dispositivo de administração de insulina para crianças diabéticas que permitisse aumentar a qualidade de vida do doente através de um produto discreto e inovador. Assim, foi estudado o histórico da doença, identificado os dispositivos existentes no mercado, levantadas as novas tecnologias no tratamento da doença e as necessidades do utilizador. $O$ fator motivador para o desenvolvimento deste projeto foi dado pela inexistência de um dispositivo de administração de insulina exclusivo para crianças e adolescentes diabéticos. O conceito do dispositivo foi baseado em uma órtese umbilical, tornando o dispositivo imperceptível no corpo do diabético em sua vida diária. Além disso, aplicação tecnológica do dispositivo permite a conexão dos dados referentes a administração de insulina via bluetooth com medidores de glicemia, telemóveis, smartphones e tablets.
\end{abstract}

Palavras-chave: Diabetes mellitus, administração de insulina, desenvolvimento de dispositivos médicos, órtese umbilical, crianças e adolescentes.

Abstract: This project aimed to develop an insulin device for diabetic children that has the capability to improve the quality of life of the patient through a discrete and innovative design. For such, it was studied the history of the disease, identified the devices available on the market, explored new technologies for the treatment of this disease and establish the necessities of the user. The motivating factor for the development of this project was given by the inexistence of an exclusive device for diabetics children and teenagers. The concept of the device was based on an umbilicus orthesis, making the device unnoticeable in the body of the diabetic during his daily life. This device is capable of sending data concerning the insulin administration via Bluetooth to blood glucose meter, cellphones, smartphone and tablets. 
Keywords: Diabetes mellitus, insulin administration, development of medical devices, umbilicus orthesis, children and teenagers.

\section{INTRODUÇÃO}

O diabetes mellitus vem aumentando consideravelmente nos últimos anos, sua incidência e prevalência atingiram mais de 382 milhões de pessoas em todo o mundo no ano de 2012, este fator corresponde a mais de $8 \%$ da população mundial (GARDETE CORREIA, 2013). O diabetes mellitus é uma doença crônica no estado hiperglicêmico crônico, podendo afetar qualquer pessoa em qualquer idade, suas causas de desenvolvimento estão ligadas a fatores genéticos e ambientais (MOREIRA; DUPAS, 2006).

O diabetes tipo 2 representa cerca de $90 \%$ do casos, enquanto que o restante é diagnosticado como diabéticos tipo 1, porém o diabetes tipo 1 é o que apresenta as maiores complicações. Por se tratar de uma doença que atinge principalmente idades que passam por uma rápida mudança biológica, física e emocional sendo necessário cuidados especiais tanto por parte médica como do doente no autocuidado, podendo interferir diretamente no desenvolvimento da criança (MINANNI, 2010). Neste caso, os diabéticos não sobrevivem sem a insulina (GARDETE CORREIA, 2010).

Existem dispositivos médicos específicos para o monitoramento e controle da doença e, como estes dispositivos estão ligados diretamente no modo de vida do utilizador, há uma constante busca por produtos que venham melhorar sua qualidade de vida e, inevitavelmente, as preocupações quanto ao preconceito associado à doença.

O maior desafio no desenvolvimento de dispositivos médicos são os estudos junto ao utilizador, essenciais para definir as características do público-alvo. Quando tratamos de crianças e adultos existe uma discrepância em termos de usabilidade, ergonomia e modo de uso, a intervenção do designer na mediação entre o desenvolvimento do projeto e o utilizador é fundamental para distinguir estas particularidades.

A inexistência de dispositivos específicos para administração de insulina para crianças e adolescentes foi motivador para o desenvolvimento deste projeto. Tornar o doente independente no autocontrole da doença é uma das grandes preocupações de médicos e agentes de saúde (MINANNI, 2010).

Este artigo consiste na apresentação do projeto desenvolvido baseado numa análise do público-alvo onde observou-se a necessidade de desenvolver um dispositivo de administração de insulina adequado com as especificidades do utilizador. Assim, o principal objetivo do dispositivo foi promover a independência no autocontrole da doença e, consequentemente, o aumento da qualidade de vida. Para tal, o projeto foi desenvolvido através de uma metodologia específica para dispositivos médicos, onde foram identificas e analisadas as necessidades do utilizador, estudadas patentes referentes a produtos deste tipo já existentes e, a partir disso, foram identificados os conceitos para a criação do produto.

\section{METODOLOGIA}

A metodologia aplicada neste projeto, Figura 1, foi desenvolvida por Santos et al. (SANTOS, 2010). A escolha desta metodologia deu-se pelo fato de ser específica para criação de dispositivos médicos. 


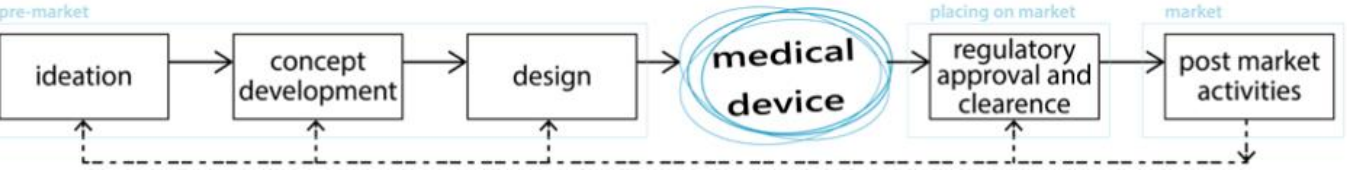

Figura 1 - Metodologia para desenvolvimento de dispositivos médicos (Santos et al.

(SANTOS, 2010))

Os dispositivos oferecidos hoje em dia tanto para o paciente adulto como criança é o mesmo, não havendo quaisquer distinções entre eles. As necessidades entre estes dois públicos é bastante diferenciada e os problemas verificados quando estes dispositivos são utilizados por crianças não atingem apenas a estética, existem fatores ergonômicos e funcionais que se diferem entre estes utilizadores. Além disso, as necessidades no tratamento da diabetes em crianças, onde em grande parte é associada a diabetes tipo 1, 0 qual é fulcral um maior número de picadas (administrações de insulina) no dia-a-dia interferindo consideravelmente na relação utilizador e dispositivo.

Devido a maior incidência da diabetes no público adulto, os dispositivos existentes no mercado acabam restringindo o desenvolvimento de dispositivos específicos para as crianças e adolescentes. Apesar de corresponderem a um menor número, cerca de $10 \%$, há uma necessidade maior de produtos específicos para o tratamento deste público (GROSSI, 2009).

Fatores ergonômicos e funcionais estão diretamente relacionados a qualidade de vida e, ainda mais, no controle da doença. Este projeto teve como objetivo a criação de um dispositivo de administração de insulina para crianças e adolescentes com diabetes tipo 1 , buscando criar um produto discreto que omitisse o aspecto medicinal com que o equipamento é visto. Desenvolver um dispositivo discreto e coerente com as particularidades nesta faixa etária, entre 10 e 14 anos, foi o principal desafio a se enfrentar.

\subsection{Identificação das necessidades}

Com base em uma pesquisa efetuada junto de Associações de Diabéticos de Portugal, onde foram realizadas pesquisas com 72 pessoas, foi possível identificar algumas necessidades que interferem na realização da administração de insulina, dando origem a um ranking das necessidades, Tabela 1 (KURT; HINTERHUBER, 1998).

\section{Tabela 1 - Ranking das necessidades dos utilizadores}

\begin{tabular}{|l|r|}
\hline Necessidade & $\begin{array}{r}\text { Citações pelo utilizador } \\
\text { (Total de 107) }\end{array}$ \\
\hline Dispositivo mais discreto & $\mathbf{1 8}$ \\
\hline Dispositivo autônomo, realizasse a medição e administração sem intervenção do diabético & $\mathbf{1 7}$ \\
\hline Dispositivo menor & $\mathbf{1 4}$ \\
\hline Mais intuitivo (prático) & $\mathbf{1 2}$ \\
\hline Indolor & $\mathbf{9}$ \\
\hline Não injetável (intranasal ou comprimido) & $\mathbf{7}$ \\
\hline Que fosse como uma bomba de infusão & $\mathbf{6}$ \\
\hline Dispositivo no interior do corpo (autônomo) & $\mathbf{5}$ \\
\hline Sem a presença de agulha & $\mathbf{4}$ \\
\hline Aspecto apelativo & $\mathbf{3}$ \\
\hline Bomba de infusão sem fio & $\mathbf{3}$ \\
\hline Necessário menos picadas & $\mathbf{3}$ \\
\hline Alertasse o nível de glicemia & $\mathbf{2}$ \\
\hline Controle por comando & $\mathbf{2}$ \\
\hline Desse para usar em qualquer parte do corpo & $\mathbf{1}$ \\
\hline Fixo no corpo & $\mathbf{1}$ \\
\hline
\end{tabular}




\subsection{Identificação análise das necessidades}

Identificadas as necessidades do utilizador e os requisitos do dispositivo, passou-se à segunda fase, desenvolvimento de conceitos, adaptada da metodologia proposta por Santo et al. (2012), Figura 2. Nesta fase são pesquisadas patentes, gerados os conceitos e seleção dos mesmos conforme as especificações do utilizador.

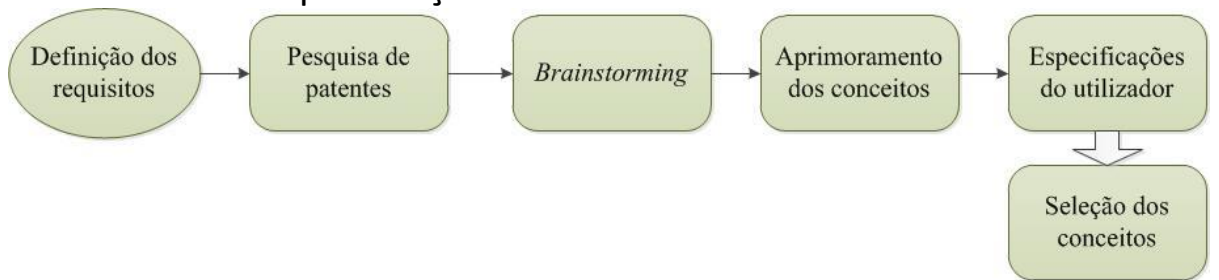

Figura 2 - Fase de geração e seleção dos conceitos (adaptado de (SANTOS, 2010))

\subsubsection{Patentes}

Para além dos dispositivos existentes no mercado, foram pesquisados produtos e tecnologias que encontram-se protegidos por patentes. Do mesmo modo, através desta pesquisa foi possível encontrar tecnologias que também podem ser aplicadas no dispositivo a ser desenvolvido.

Através da plataforma Google Patentes foram analisadas 79 patentes que pudessem ajudar no desenvolvimento de um novo dispositivo. O processo de busca por patentes é descrito na Figura 3.

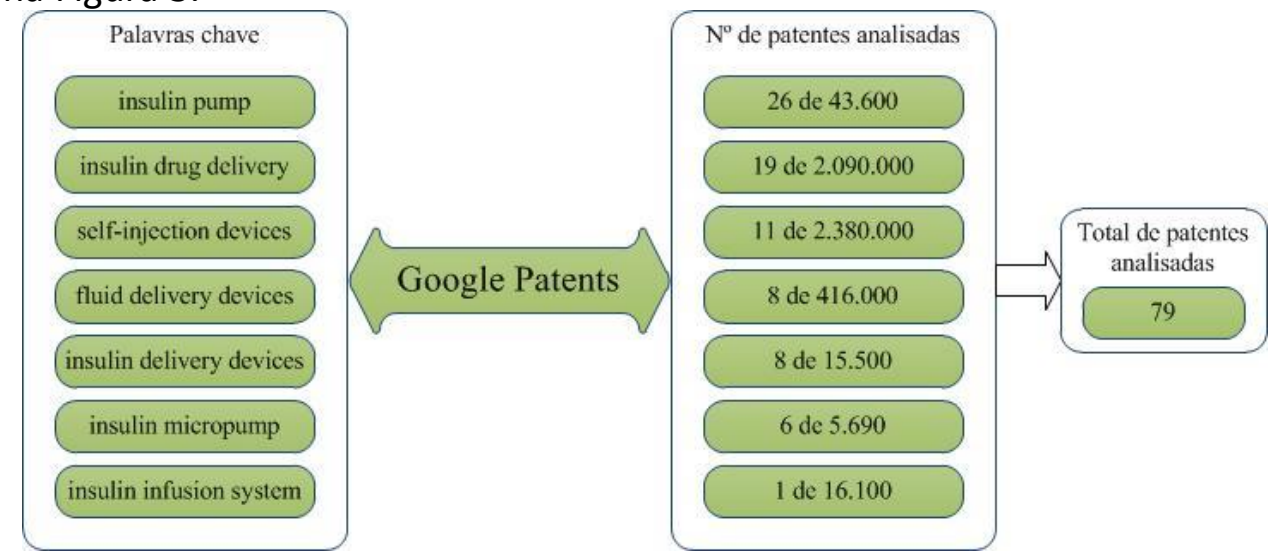

Figura 3 - Recolha e seleção de patentes

\subsubsection{Brainstorming}

Caracterizada como a fase da "tempestade de ideias", o brainstorming é a melhor ferramenta para iniciar a geração de conceitos de um produto. Aqui são apresentadas possíveis ou impossíveis soluções para resolver as necessidades encontradas (GOMES, 2011). Foram gerados três conceitos com sistemas de funcionamento distintos, apenas um foi selecionado para geração de novos conceitos.

O primeiro conceito, Figura 4, é um dispositivo fixo no corpo, por um material adesivo, durante o dia, podendo ser aplicado no braço, perna ou barriga, de material flexível e no seu interior contém um reservatório de insulina. No topo seriam feitas marcações, em unidades de insulina, para controlar o nível de insulina administrada. A insulina seria injetada utilizando uma cânula, que seria encaixado no compartimento principal (reservatório). Numa das extremidades do reservatório teria uma saliência contendo insulina, que quando pressionada injetaria a insulina no subcutâneo. A principal vantagem é o tamanho, o custo e praticidade do dispositivo. Por outro lado, a principal desvantagem é a 
precisão da dose administrada e a possível administração de insulina não intencional, por acidente.

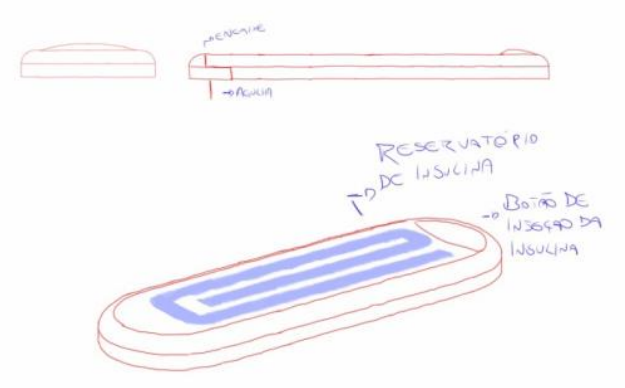

Figura 4 - Conceito de um dispositivo adesivo

O segundo conceito, Figura 5, seria um dispositivo que administrasse insulina por laser, funcionando como uma caneta. Ao pressionar uma das extremidades seria liberada insulina em forma de cosmético e ao mesmo tempo acionaria o laser para o medicamento penetrar na pele. A tecnologia empregada faria uso do sistema descoberto na Universidade de Coimbra, Portugal (LaserLeap). Como desvantagem tem-se a inexistência de uma insulina compatível com esta tecnologia.

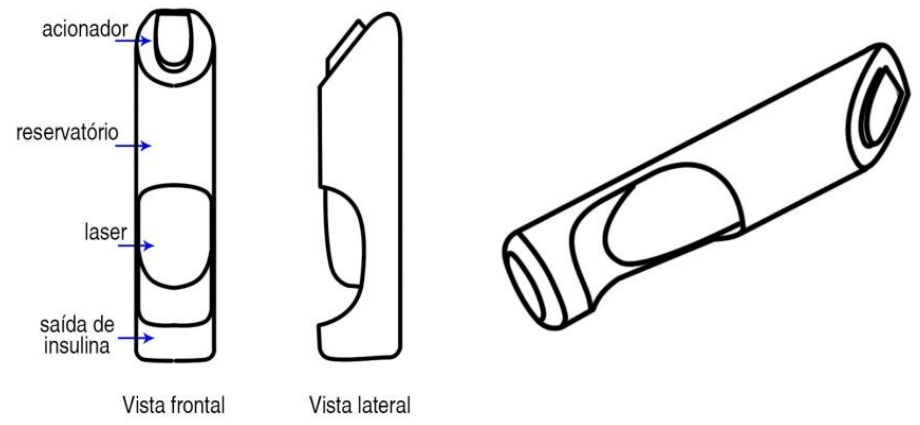

Figura 5 - Conceito de uma caneta de insulina a laser

Por fim, o último conceito, Figura 6, seria uma mini bomba de infusão para umbigo. Formada por duas partes, a primeira consiste no suporte da agulha para fixar no umbigo, na parte externa o material seria flexível ou com memória de forma para poder ser inserido no umbigo e depois expandisse para não se mover. A segunda parte seria o reservatório de insulina, podendo ser retirada para recarregar com o líquido. Contaria com um sistema para transmissão de dados sem fio, passando informações da quantidade de insulina no reservatório, gráficos para controle e também o registro para futuras informações solicitadas pelo médico. Sua aplicação é bastante próxima de uma bomba de insulina, ou seja, seria inserido o suporte no umbigo e depois encaixado o reservatório. A principal vantagem seria a discrição na administração de insulina, liberdade de movimentos e o controle rigoroso das doses. Este sistema foi selecionado para prosseguir na geração de novos conceitos.

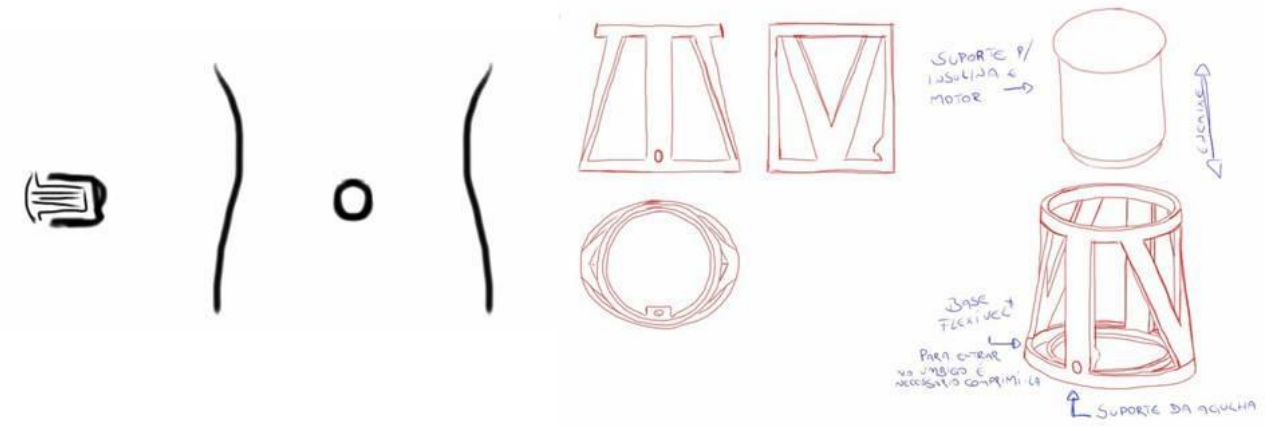

Figura 6 - Conceito de uma mini bomba de infusão 


\subsubsection{Seleção de conceitos}

Analisadas as necessidades do utilizador e as patentes, deu-se continuidade a geração de conceitos a partir do conceito definido no brainstorming. Num primeiro instante foi empregado o desenho gráfico manual em papel, evitando assim o bloqueio da criatividade e melhor expressão das ideias (GOMES, 2011). Como fonte de inspiração dos conceitos utilizou-se o conceito de uma órtese umbilical, da marca Ortho Pauher, trata-se de um dispositivo para modelar o umbigo pós-cirurgia, fabricado em silicone (sem informação das dimensões do produto).

Para mais, todos os conceitos foram desenvolvidos com base na tecnologia de uma nano bomba de infusão, desenvolvida pela Debiotech baseados em chips MEMS (MicroElectro-Mechanical Systems), Figura 7, descrita na Patente EP2469089 A1. Trata-se de uma bomba segura, pequena, precisa, confiável e de baixo custo de fabricação, permitindo a aplicação até mesmo em dispositivos descartáveis, e encontra-se em constante desenvolvimento (DEBIOTECH, 2013).

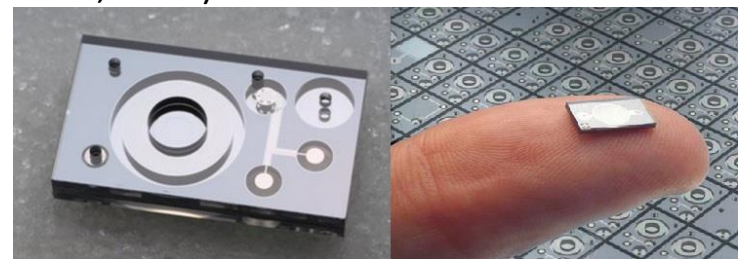

Figura 7 - Nano bomba de infusão baseada em chips MEMS (adaptado de (DEBIOTECH, 2013))

Os conceitos desenvolvidos (Anexo I) foram selecionados e avaliados entre si, a avaliação foi feita mediante os requisitos do projeto e as necessidades do utilizador. Para realizar esta tarefa foi aplicada a ferramenta Concept Screening, que possibilita esta comparação através de uma pontuação, sendo aqui " +1 " positivo, " 0 " neutro e " 1 " negativo, Tabela 2 (ULRICH, 2004). 
Tabela 2 - Tabela de avaliação e seleção dos conceitos

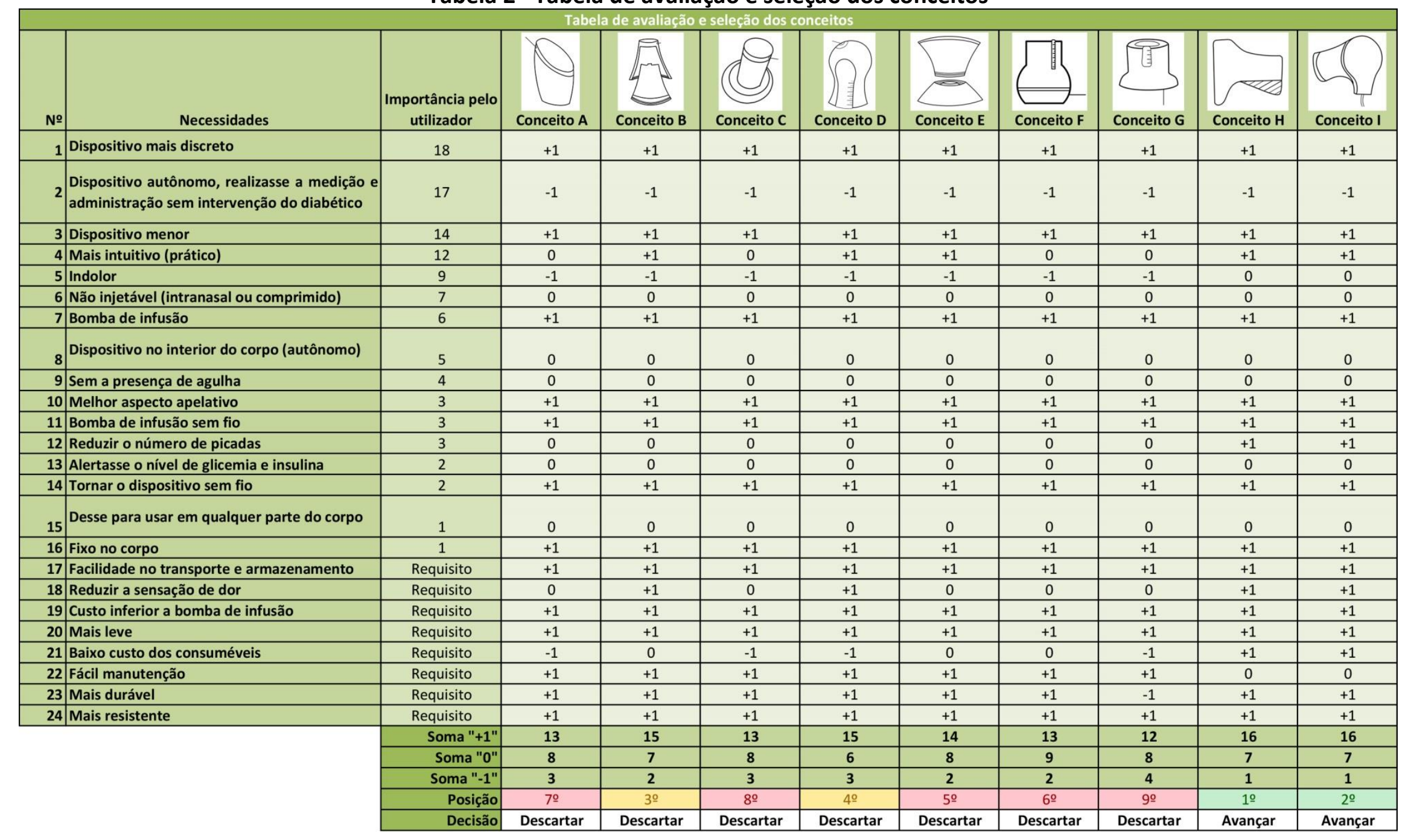


Avaliados os conceitos perante as necessidades dos utilizadores e os requisitos do dispositivo, destacaram-se os conceitos $\mathrm{H}$ e I para originarem o dispositivo pretendido. Apesar da semelhança entre os dois conceitos, o principal diferencial está no modo de fixação no corpo. Por um lado tem-se um dispositivo que não necessita aplicação de outro material no processo de fabricação para aderir à parede umbilical. Por outro, tem-se um dispositivo fabricado a partir de dois materiais com propriedades diferentes e que dependendo das características podem se adaptar melhor nesta parte do corpo. Esta distinção é crucial no desenvolvimento do dispositivo, pois aspectos como conforto, limitações dos movimentos e fatores externos como umidade e suor poderão afetar o desempenho do dispositivo. Seguindo neste pensamento, foram combinados os dois conceitos para criação do dispositivo final, apresentado na fase seguinte.

\subsubsection{Projeto técnico construtivo}

Esta etapa de desenvolvimento do projeto corresponde a fase de Design da metodologia adotada, Figura 8. A fase de Design corresponde no desenvolvimento técnico do dispositivo, incluído detalhes de sua tecnologia e construção.

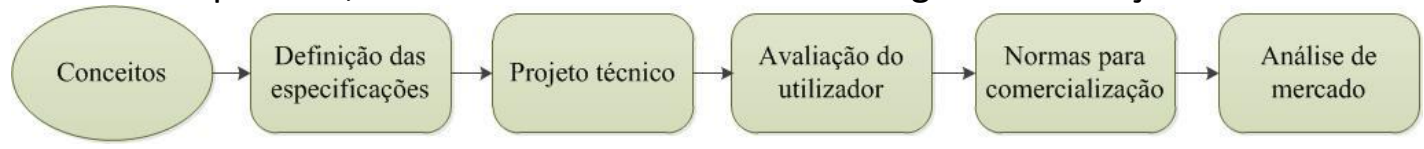

Figura 8 - Fase de Design (adaptado de (SANTOS, 2010))

Primeiramente, realizou-se uma pequena avaliação para dimensionar o umbigo das crianças. Para isso, foram abordadas 10 crianças para verificação das dimensões do umbigo, Tabela 3.

Tabela 3 - Tabela das dimensões dos umbigos analisados

\begin{tabular}{|c|c|c|c|c|c|c|}
\hline & Idade & Sexo & Profundidade (P) & Diâmetro (D) & Diâmetro expandido & \% de dilatação \\
\hline Criança 1 & 10 & $\mathrm{~F}$ & 8,5 & 7,5 & 10 & $25 \%$ \\
\hline Criança 2 & 10 & $M$ & 10,7 & 9,5 & 12,5 & $30 \%$ \\
\hline Criança 3 & 10 & $M$ & 11,3 & 10 & 12 & $20 \%$ \\
\hline Criança 4 & 11 & $\mathrm{~F}$ & 11 & 9 & 12,5 & $35 \%$ \\
\hline Criança 5 & 12 & $M$ & 14 & 11,3 & 13,5 & $22 \%$ \\
\hline Criança 6 & 12 & $\mathrm{~F}$ & 13,5 & 12 & 14 & $20 \%$ \\
\hline Criança 7 & 13 & $\mathrm{~F}$ & 15 & 10 & 13 & $30 \%$ \\
\hline Criança 8 & 14 & M & 20 & 15 & 20 & $50 \%$ \\
\hline Criança 9 & 14 & M & 17 & 12,5 & 16 & $35 \%$ \\
\hline Criança 10 & 12 & $M$ & \multicolumn{4}{|c|}{ fora dos padrões normais } \\
\hline Média & 11,8 & & 13,4 & 10,8 & 13,7 & $30 \%$ \\
\hline
\end{tabular}

A Figura 9 representa os critérios de medição dos umbigos. Foi considerado umbigo normal aquele que apresenta uma profundidade entre a gola e a cicatriz umbilical.

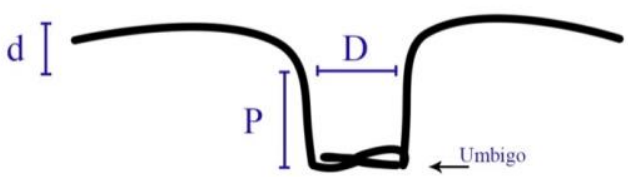

Figura 9 - Padrão das medições das dimensões do umbigo

A partir desta análise delimitou-se que o dispositivo deveria ter as seguintes dimensões: diâmetro (D) mínimo de 10 milímetros $(\mathrm{mm})$ e máximo de $15 \mathrm{~mm}$; e profundidade mínima de $15 \mathrm{~mm}$. 
Escolhido o conceito final e analisadas as especificações dos componentes vitais para o funcionamento do dispositivo, desenvolveu-se o modelo CAD 3D através do software SolidEdge ST5. Através desta ferramenta 3D foi possível visualizar o dispositivo nas dimensões reais, a apresentação detalhada do projeto e a simulação de vistas realistas do produto.

O dispositivo é dividido em dois conjuntos, o conjunto principal contém todos os componentes para controle e bombeamento da insulina, o outro é o conjunto de infusão, descartável, que ligará a bomba de infusão ao subcutâneo, Figura 10.

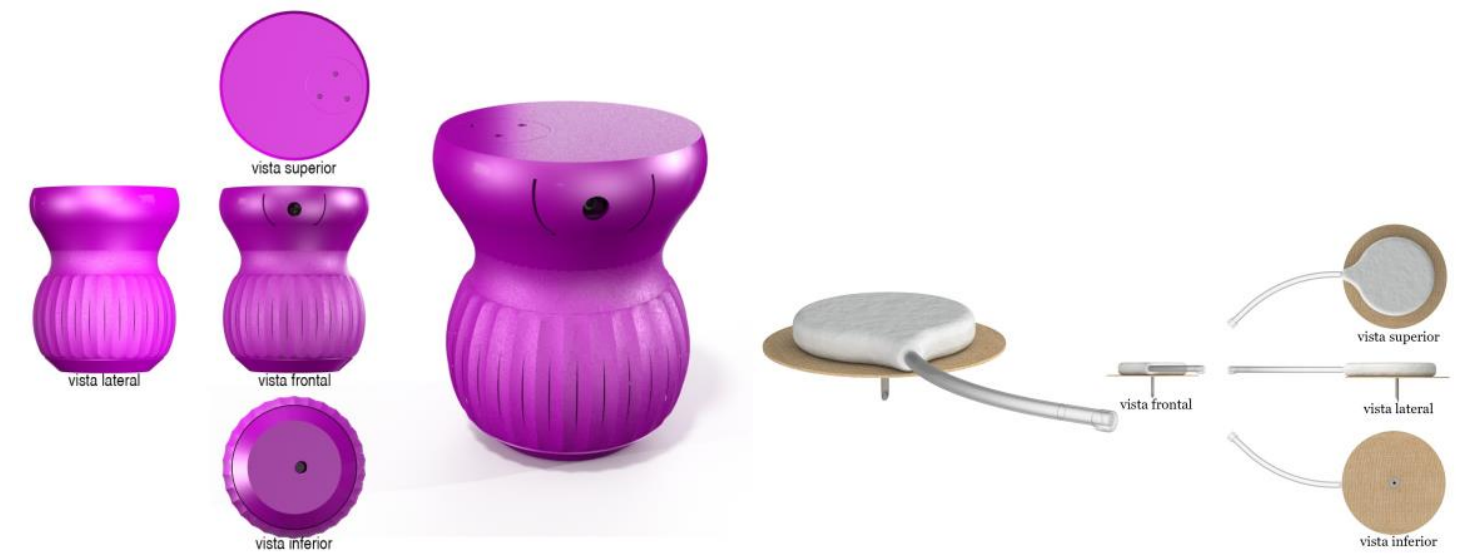

Figura 10 - Vistas do conjunto principal do dispositivo (E) e Conjunto de infusão (D)

O formato do dispositivo foi pensado como um produto simples, esteticamente agradável, confortável, tecnicamente funcional, que priorizasse a discrição durante a administração de insulina e que fosse facilmente manuseado pelas crianças. A fabricação do dispositivo permite vedação total dos componentes, viabilizando o exercício de atividades como nadar sem prejudicar seu funcionamento.

O dispositivo é inserido no umbigo da seguinte forma, Figura 11:

1) segurar o dispositivo e pressionar a espuma com memória de forma até o final;

2) girar o dispositivo enquanto pressiona a espuma com memória de forma;

3) inverter e introduzir o dispositivo no umbigo.

Desta forma o dispositivo entra com maior facilidade no umbigo.
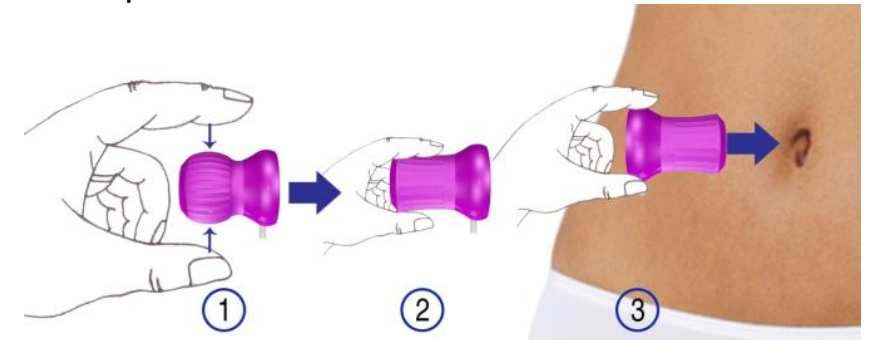

Figura 11 - Fases da inserção do dispositivo no umbigo

Após inserido o dispositivo no umbigo a espuma com memória de forma retornará ao seu formato original, tomando a forma do umbigo e mantendo pressão para que o dispositivo não seja removido com tanta facilidade.

O próximo passo passa por fazer a ligação do conjunto de infusão e a bomba de infusão, Figura 12. Para isso, basta ligar o conector do conjunto de infusão ao conjunto principal, a ligação entre os dois conjuntos é por meio de um sistema de encaixe. 


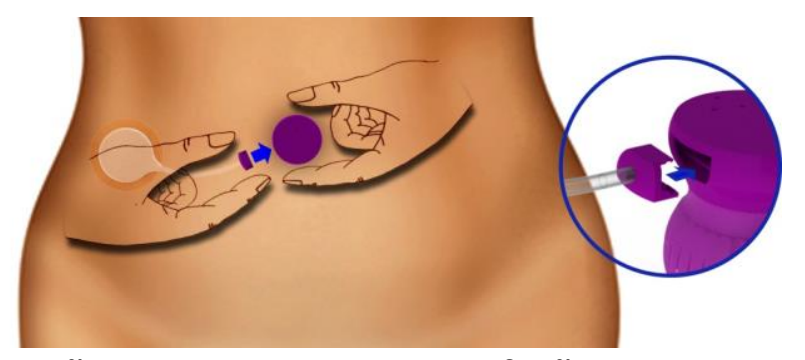

Figura 12 - Conexão entre o conjunto de infusão e a bomba de insulina

Por fim, é preciso inserir a cânula no subcutâneo. Realizadas todas estas etapas dá-se início ao bombeamento de insulina, a insulina passa pela câmara de bombeamento da nano bomba de infusão, percorre o tudo do conjunto de infusão e é administrada no subcutâneo pela cânula.

O controle do dispositivo poderá ser realizado por meio dos dispositivos agregados, ou seja tecnologias que venham a ser compatíveis com este produto como smartphones, tablets e medidores de glicemia com tecnologia bluetooth. Para melhor compreensão do funcionamento do controle do dispositivo apresenta-se o fluxograma da Figura 13.
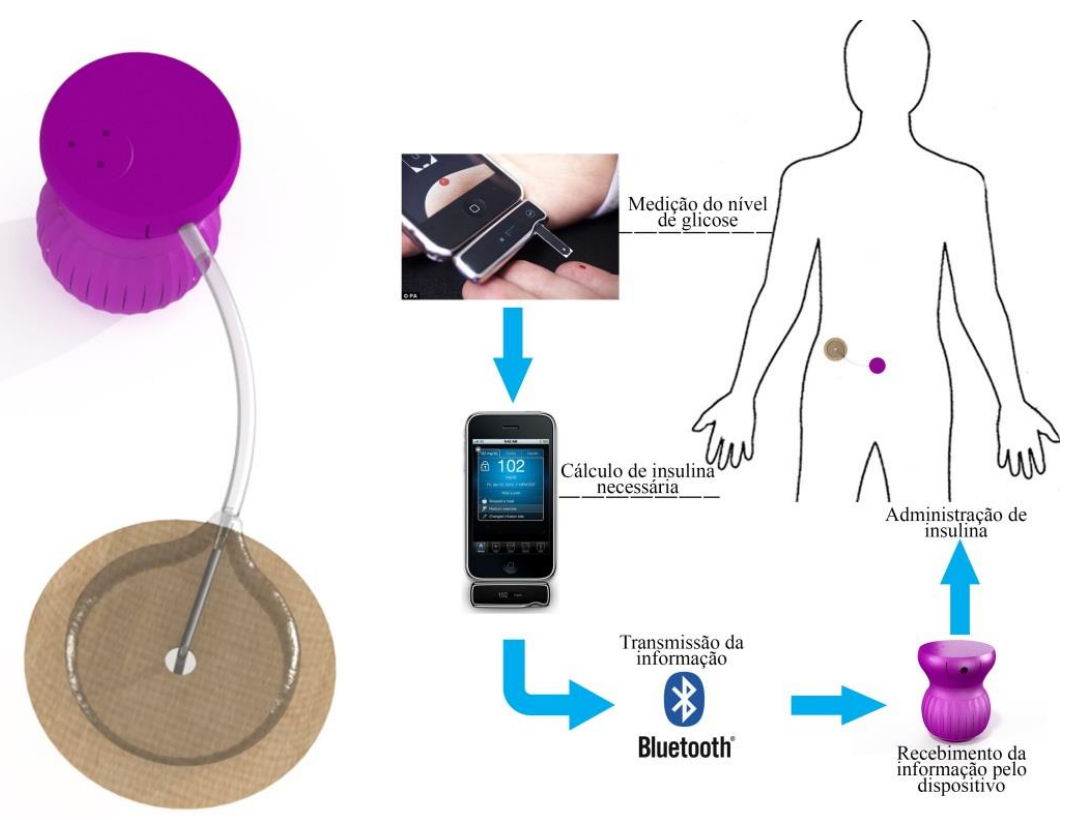

Figura 13 - Fluxograma do funcionamento do dispositivo

Devido ao público alvo estar ligado a diversas mudanças e o formato bastante simplificado do produto sugere-se algumas cores que o dispositivo possa ser fabricado, Figura 14. Deste modo, poderão ser atingidos os vários gostos das crianças diabéticas.

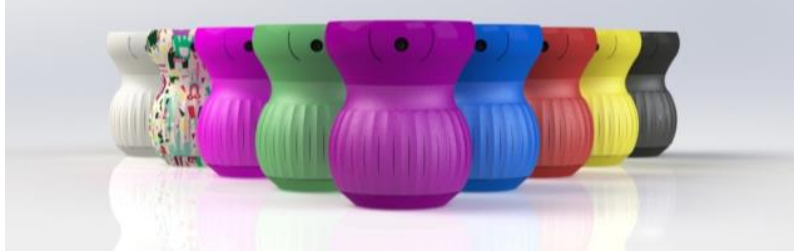

Figura 14 - Sugestões de cores para produção do dispositivo

\subsubsection{Análise de mercado}

A avaliação com o utilizador deu-se pela realização de um inquérito através do Google Form, por não obter protótipo teste apresentaram-se imagens realistas do 
conceito e postas perguntas para avaliar a sensação que o diabético tinha analisando as referências fotográficas. O inquérito foi distribuído na Associação de Jovens Diabéticos de Portugal e ficou disponível por 3 semanas, apenas 6 diabéticos responderam ao inquérito, Figura 15.

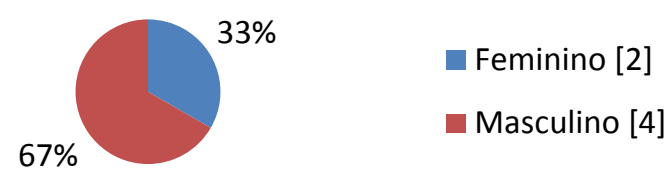

Figura 15 - Gênero dos diabéticos inquiridos

As idades dos inquiridos dificultaram uma análise mais profunda e concreta sobre a aceitação do produto, todos os inquiridos tinham mais de 20 anos de idade, interferindo na análise entre o público alvo que o produto se destina, crianças diabéticas entre 10 e 14 anos. Foi questionado se já havia conhecimento sobre a tecnologia da nano bomba de infusão e se já conheciam algum dispositivo que empregava esta tecnologia, todos responderam que não conheciam nem a tecnologia tampouco algum dispositivo.

Para verificar o emprego do sistema Bluetooth no dispositivo havia uma pergunta se o diabético portava algum dispositivo que tinha este sistema de transmissão de dados, todos responderam que sim. Também, para analisar a satisfação de controlar as administrações de insulina por algum destes dispositivos as respostas foram, Figura 16.

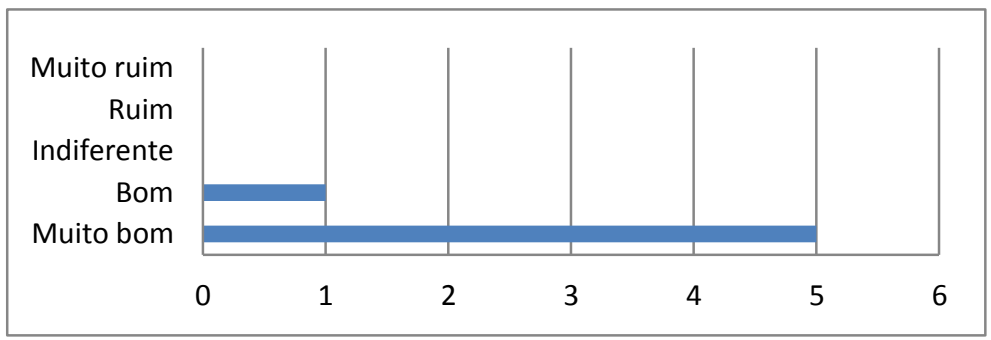

Figura 16 - Classificação do emprego de controle do dispositivo sem fio

Outra questão era referente ao grau de satisfação por ter um dispositivo ligado ao corpo que administrasse insulina mesmo quando estivesse a praticar atividades físicas, Figura 17.

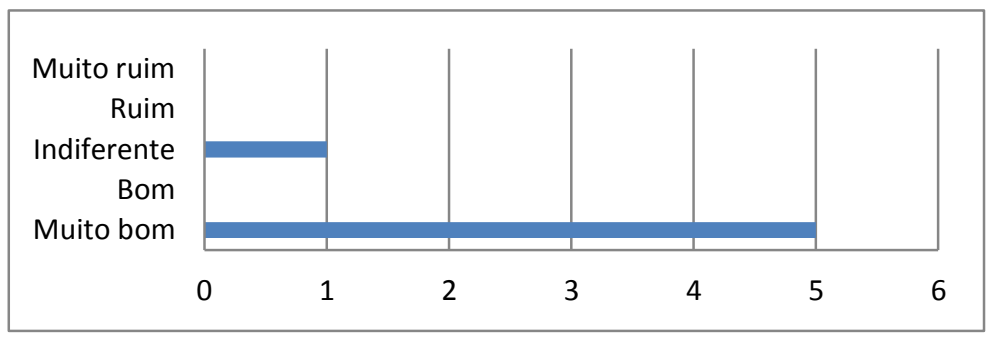

Figura 17 - Satisfação em administrar insulina mesmo quando está a praticar atividades físicas

A discrição do produto durante a administração teve uma boa classificação, entre muito discreto, discreto, indiferente, indiscreto e muito indiscreto, recebeu todas as resposta considerando o produto discreto. Esta avaliação atrapalha o desempenho dos resultados por não ter um dispositivo protótipo, entretanto a classificação percebida foi considerada boa. Outro ponto que possa ter sido 
influenciado pela falta de um dispositivo teste foi em relação ao conforto do dispositivo percebido pelas imagens, Figura 18.

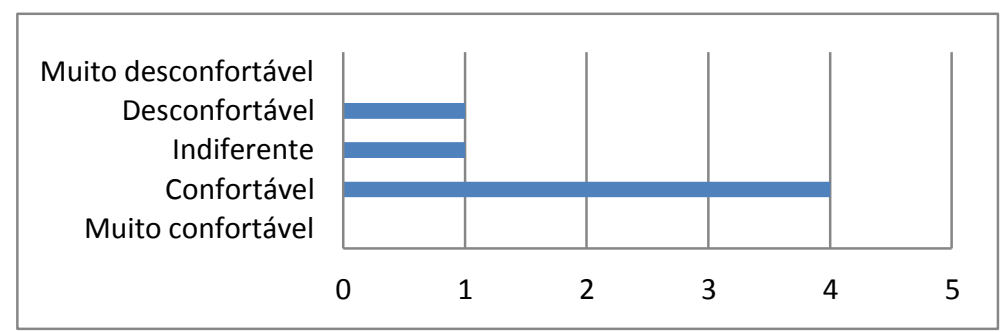

Figura 18 - Grau de avaliação do conforto percebido no dispositivo

Os diabéticos foram questionados se sua qualidade de vida poderia mudar com o uso do dispositivo, Figura 19.

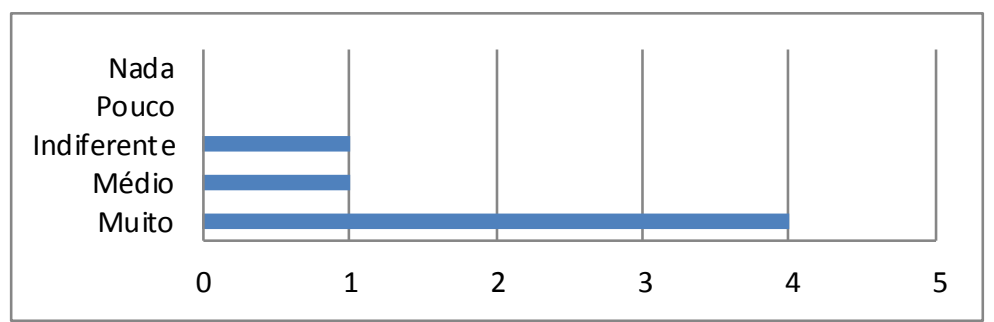

Figura 19 - Melhora da qualidade de vida com o uso do dispositivo

Por fim, para encaixar o produto no mercado foi questionado quanto o diabético estava disposto a pagar pelo dispositivo, Figura 20. Através desta questão é possível estabelecer um custo estimado que os diabéticos estivessem dispostos a pagar pelo dispositivo.

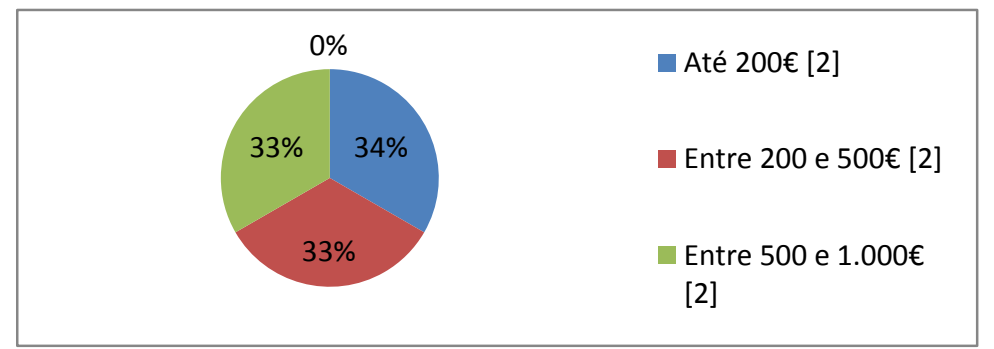

Figura 20 - Custo que o diabético estaria disposto a pagar pelo dispositivo

\section{CONCLUSÃO}

O presente projeto centrou-se no desenvolvimento de um dispositivo de administração de insulina para crianças diabéticas, fazendo uso de uma metodologia específica para dispositivos médicos. A metodologia tornou-se um guia para não deixar escapar quaisquer elementos importantes no desenvolvimento de um produto médico, assegurando que todas as fases do projeto fossem cumpridas com eficácia.

O principal objetivo cumprido neste projeto foi desenvolver um dispositivo que melhorasse a qualidade de vida de seus utilizadores, as crianças diabéticas, que vêm crescendo consideravelmente nos últimos anos. Através de um dispositivo discreto, pequeno e coerente com as peculiaridades características das crianças entre 10 e 14 anos eliminou-se o aspecto de "doença" que os dispositivos atuais no mercado eram vistos. Ainda mais, o dispositivo desenvolvido reduz as limitações impostas pelos atuais dispositivos grandes e indiscretos, como a prática esportiva sem restrições ou incômodos. 
Basicamente, o projeto foi dividido em três grandes fases, a recolha de informações acerca da doença, a pesquisa com utilizador e o desenvolvimento do dispositivo. Na fase de identificação das necessidades, o contato direto com os doentes possibilitou mais-valia para identificação das suas limitações e a vivência durante o dia entre o diabético e seu tratamento. Num todo, esta análise permitiu identificar claramente a ausência de um dispositivo menor e mais discreto para administração de insulina.

Por fim, desenvolveu-se um dispositivo para administração de insulina para crianças diabéticas, onde a pesquisa de novas tecnologias ajudou a identificar uma nano bomba de infusão para aplicação no dispositivo. Com isso, chegou-se ao conceito de um pequeno dispositivo para ser usado no umbigo, tornando-se invisível embaixo das roupas e melhorando a qualidade de vida dos utilizadores.

Como perspectivas futuras, o desenvolvimento deste trabalho gerou 0 interesse em algumas propostas como:

- Aprimoramento do projecto CAD conforme as especificações do fabricante da nano bomba de infusão;

- O desenvolvimento de um dispositivo protótipo para análise do utilizador, verificando sua usabilidade, conforto e precisão nas administrações. Além disso, analisar a melhora da qualidade de vida dos diabéticos comparado com outros dispositivos;

- Recolher uma amostra maior de diabéticos para análise do mercado;

- Criar um aplicativo para o controle de administração de insulina próprio para o dispositivo;

- Desenvolver um dispositivo destinado ao público adulto e/ou diabéticos tipo 2;

- Desenvolver uma caneta de insulina que permita a mistura de diversas insulinas.

\section{REFERÊNCIAS}

BELLIS, Mary. Hypodermic Needle - Syringe Needle. Inventors.about.com. (s/ data). Disponível em <http://inventors.about.com/library/inventors/blsyringe.htm>. Acesso em: 31 Jan. 2013.

Bombas de Infusão. Projeto Inovador, 2008. Disponível em < http://projetoinovador.spaceblog.com.br/103580/Bombas-de-Infusao-de-Insulina/>. Acesso em: 29 Abr. 2013.

DEBIOTECH. Nanopump. Debiotech S.A. Switzerlan, 2013. Diponível em < http://www.debiotech.com/products/msys/chronojet.html>. Acesso em: 12 Mar. 2013.

DIABETES-SUPPORT. The history of insulin pumps. Disponivel em < http://www.diabetes-support.org.uk/info/?p=287>. Acesso em: 01 Fev. 2013.

DUARTE, Rui. Diabetologia Clínica. 3ạ Edição. Lisboa: Lidel, 2002.

GARDETE CORREIA, Luís et. al. Diabetes: Factos e números 2013. Relatório Anual do Observatório Nacional de Diabetes. Lisboa: Sociedade Portuguesa de Diabetologia, 2013.

GOMES, Luiz Antônio Vidal de Negreiros. Criatividade e design: um livro de desenho industrial para projeto de produto. Porto Alegre: sCHDs, 2011. 
GROSSI, Sonia Aurora Alves; PASCALI, Paula Maria. Cuidados de enfermagem em diabetes mellitus. Manual de Enfermagem. São Paulo: Sociedade Brasileira de Diabetes, 2009.

KURT, Matzler; HINTERHUBER, Hans H.. How to make product development projects more successful by integrating Kano's model of customer satisfaction into quality function deployment. Technovation, v.18, n1, 25-38. Jan, 1998.

LIBERATORE JR., Raphael Del Roio; DAMIANI, Durval. Bomba de infusão de insulina em diabetes melito tipo 1. Jornal de Pediatria, Porto Alegre, v.82, n.4, 249254, Ago. 2006.

2013.

MEDTRONIC. Medtronic Quick-set: conjunto de infusão. Medtronicdiabetes, http://www.medtronicdiabetes.com/sfc/servlet.shepherd/version/download/068C000 0000KFXb>. Acesso em: 20 Jun. 2013.

MINANNI, Carlos André et. al. Abordagem integral do adolescente com diabetes. Adolescência e Saúde. 2010;7(1): 45-52.

MOREIRA, Patrícia Luciana; DUPAS, Giselle. Vivendo com o diabetes: a experiência contada pela criança. Revista Latino-Americana de Enfermagem, Ribeirão Preto, v.14, n.1, Feb. $2006 . \quad$ Disponível em <http://www.scielo.br/scielo.php?script=sci_arttext\&pid=S0104$11692006000100004 \&$ Ing=en\&nrm=iso>. Acesso em 20 Jan. 2013.

NOVO NORDISK. Novo Nordisk History. Bagsværd, 2011.

OLIVEIRA, Maria Camargo de. Preparo e aplicação de insulina sem mistério. São Paulo: Centro BD de Educação em Diabetes, 2011.

Órtese macia para umbigo. Casa do Enfermeiro. Solostocks, 2013. Disponível em <http://www.solostocks.com.br/venda-produtos/saude-medicina-beleza/outrossaude-medicina-beleza/ortese-macia-para-umbigo-1372134>. Acesso em: 18 Fev. 2013.

SANTOS, Isa C. T. Product development methodologies: the case of medical devices. 2012. Thesis submitted to the Faculdade de Engenharia da Universidade do Porto for the degree of Doctor of Philosophy in Leaders for Technical Industries of the MIT - Portugal Program.

SCIENCE MUSEUM. Hipodermic syringe. Science \& Society Picture Library. 2003.

STEWART, Kristine M. et. al. Insulin Delivery Devices. Journal of Pharmacy Practice. New York, v.17, no1, 20-28. 2004.

ULRICH, Karl T.. Product design and development. Universidade da Califórnia, 2004.

TESCKE, Lucas R. et al. Desenvolvimento de um dispositivo de administração de insulina: o caso das crianças diabéticas. ISBN-13: 978-3-639-61793-1, ISBN-10: 3639617932, EAN: 9783639617931. Saarbrücken (Alemanha): Novas Edições Acadêmicas, 2014.

\section{ANEXOS}

Conceito A - O sistema do conceito A, Figura 21, funciona por meio de uma agulha que é pressionada através de uma mola para penetrar no subcutâneo logo após que o dispositivo é inserido no umbigo. O compartimento da agulha é descartável e substituído dentre 3 e 5 dias, seu encaixe em "L" permite facilidade e rapidez na troca 
da agulha. A aderência as paredes do umbigo é feita pelo material e textura do material empregado, além da agulha já proporcionar a base da fixação. Seu formato anatômico proporciona facilidade ao inserir o dispositivo no umbigo e discrição durante a administração de insulina.

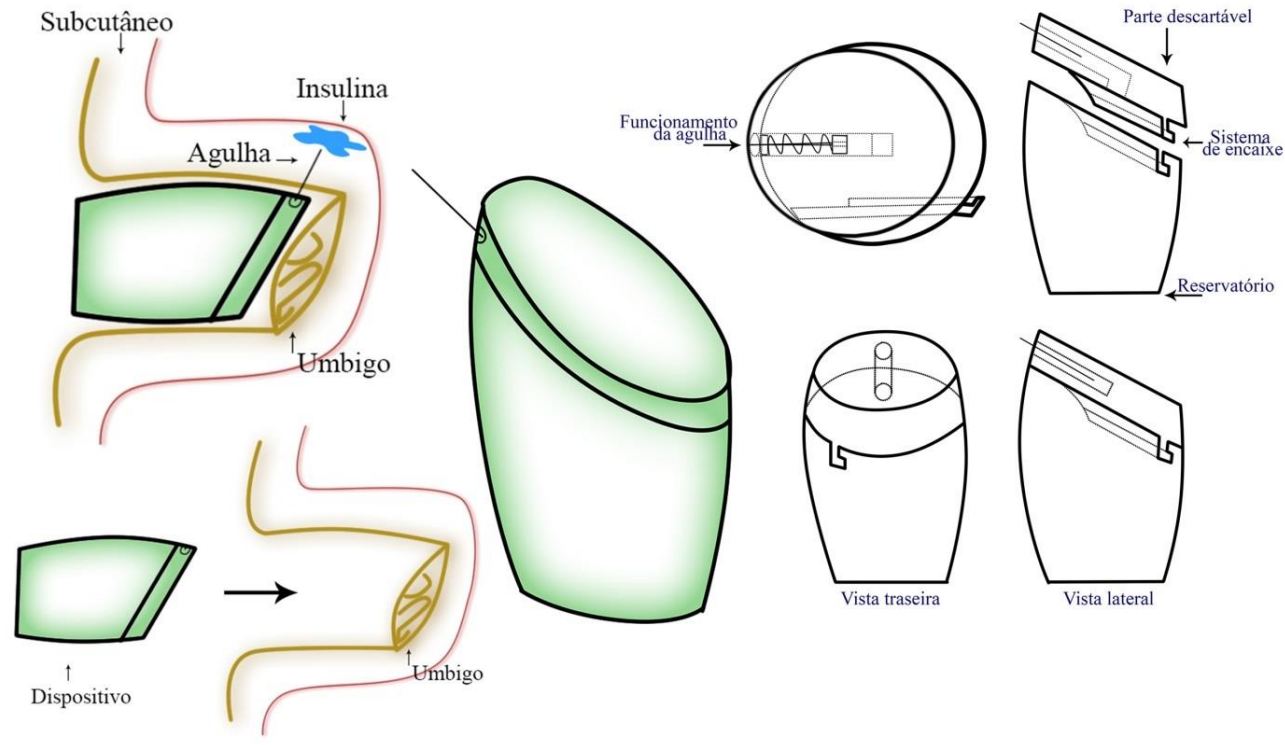

Figura 21 - Conjunto de imagens do conceito $A$

Conceito B - Neste outro conceito, Figura 22, há um suporte que é inserido primeiramente no umbigo, em seguida é rosqueado o compartimento principal, que contém insulina, bomba e bateria. O suporte é composto por uma agulha e ao inserir e girar o reservatório, por meio de um sistema mecânico, a agulha penetra no subcutâneo.

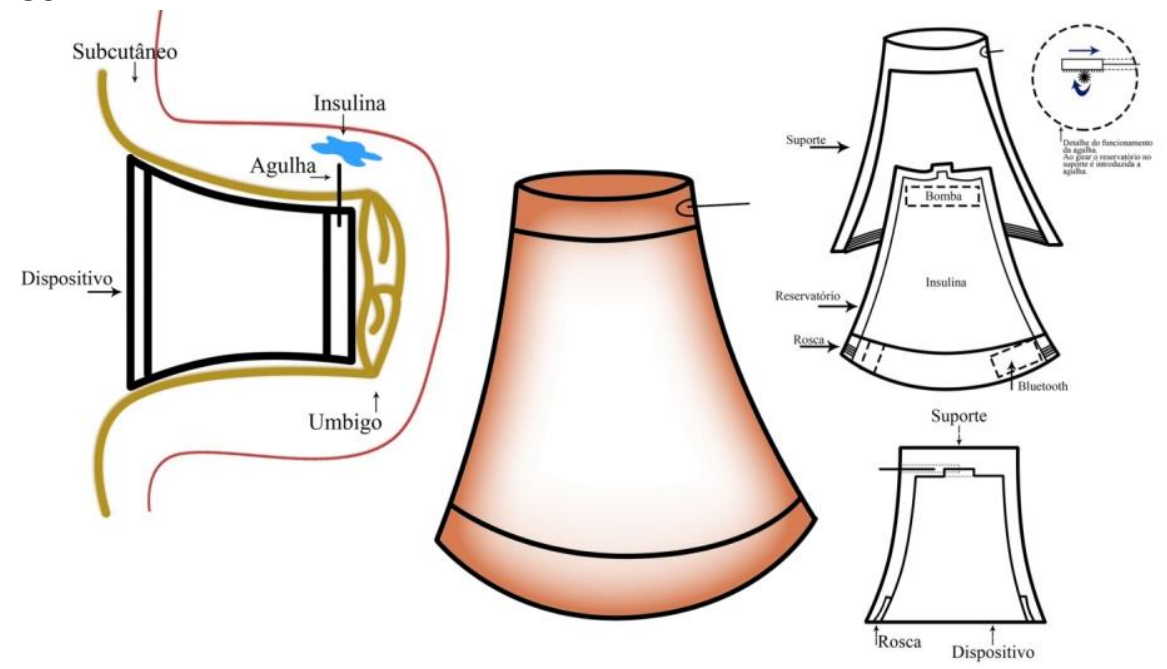

Figura 22 - Conjunto de imagens do conceito $B$

Conceito C - O conceito C, Figura 23, tem seu sistema de funcionamento bastante aproximado do conceito $\mathrm{A}$. A agulha penetra no subcutâneo por meio de uma mola assim que o dispositivo é inserido no umbigo. $O$ dispositivo é fixo através de um adesivo dupla face descartável e trocado a cada utilização. 


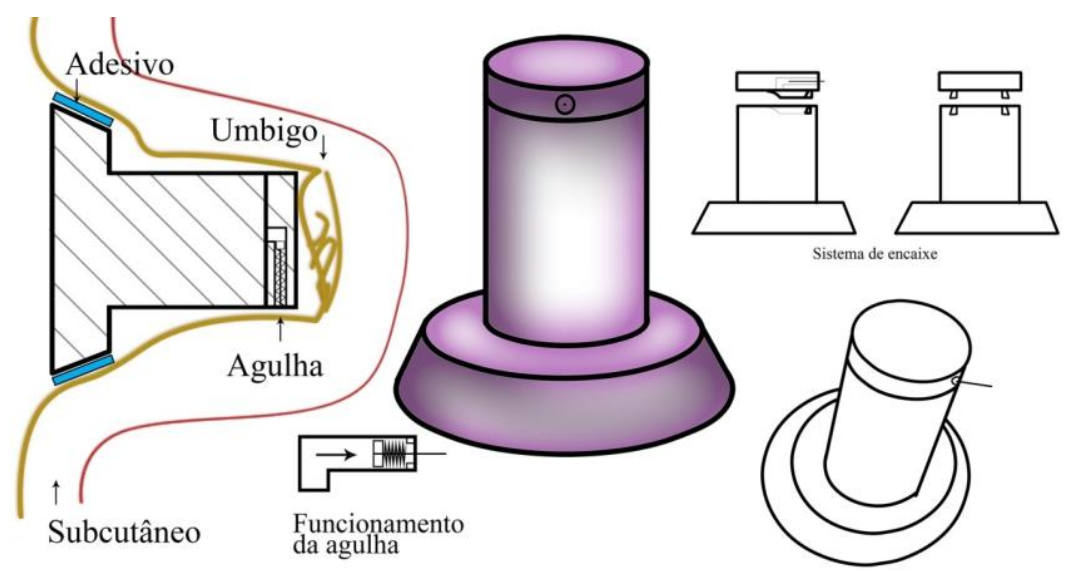

Figura 23 - Conjunto de imagens do conceito $C$

Conceito D - No conceito D, Figura 24, intenta representar um suporte colocado anteriormente no umbigo e um reservatório de insulina contendo bomba, bateria e sistema de transmissão de dados. O suporte fabricado de material rígido e seu formato anatômico torna sua fixação sem necessidade de adesivos. Neste caso também é usado o sistema de mola para penetrar a agulha no subcutâneo. O compartimento principal é encaixado no suporte, que já se encontra dentro do umbigo. Além disso, o suporte é descartável e trocado de 3 a 5 dias.
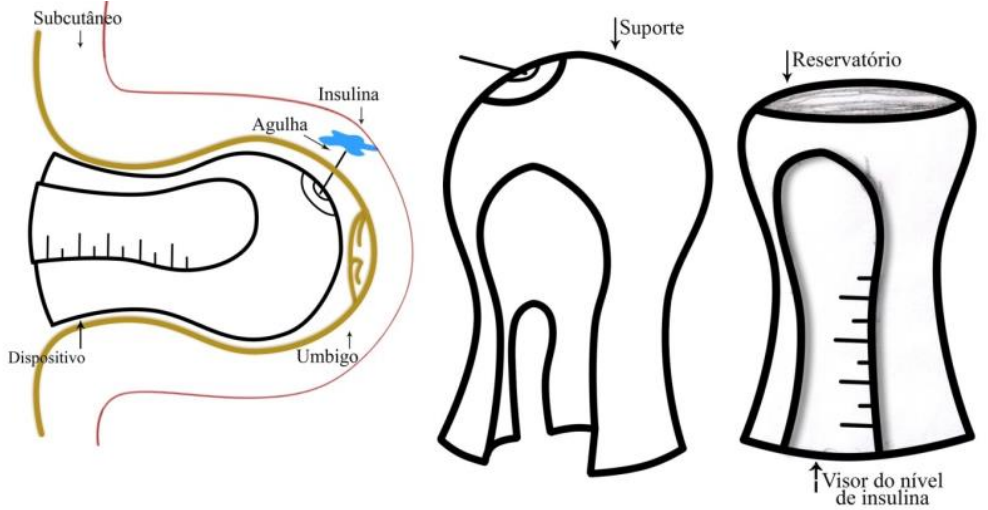

Figura 24 - Conjunto de imagens do conceito D

Conceito E - Como o conceito anterior, o único diferencial deste, Figura 25, é seu suporte em polímero flexível. Seu encaixe é feito por rosca. Deste modo, reduziriam os custos do suporte.

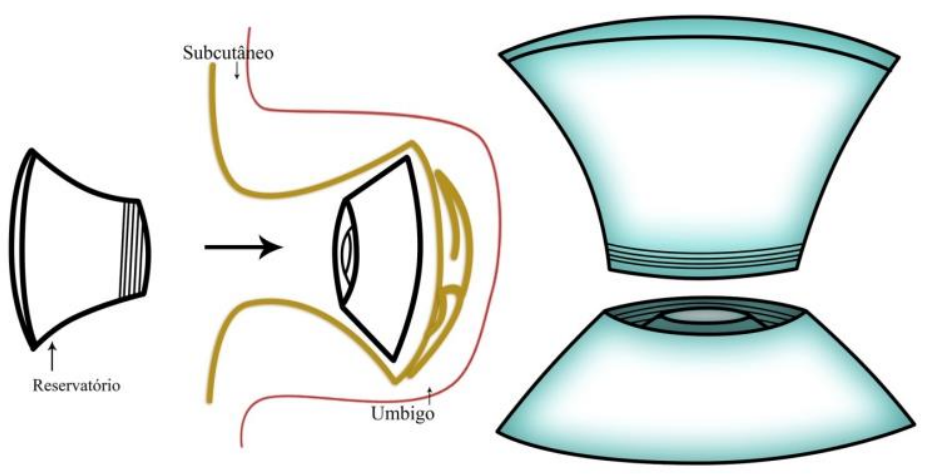

Figura 25 - Conjunto de imagens do conceito $\mathrm{E}$

Conceito F - Este conceito, Figura 26, funciona basicamente como o conceito A. Seu diferencial é o formato anatómico fixando o dispositivo com mais facilidade no umbigo. 

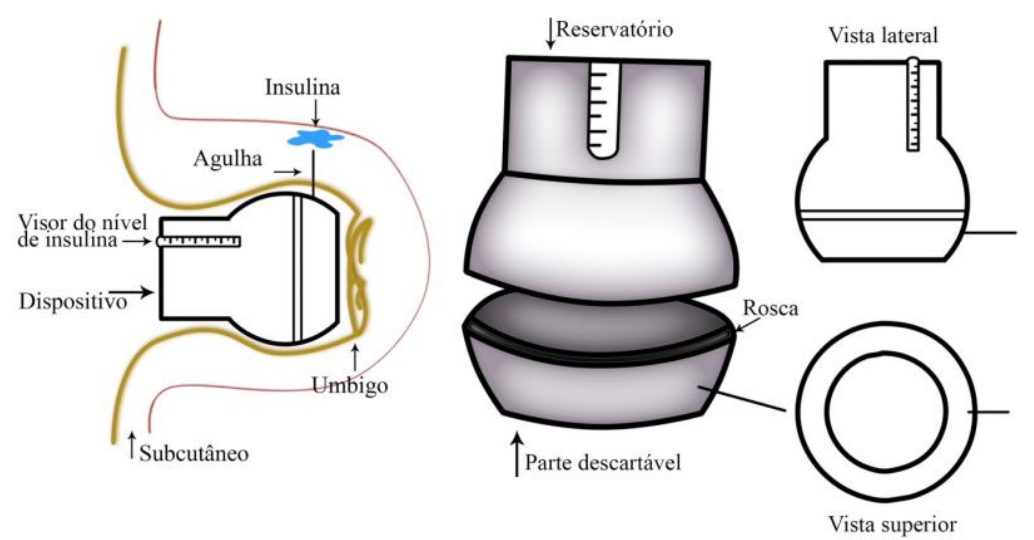

Figura 26 - Conjunto de imagens do conceito $\mathrm{F}$

Conceito G - O conceito G, Figura 27, consiste em um sistema com troca de agulha. Sendo assim, não há necessidade de remover o dispositivo para realizar a troca de agulha, apenas o reabastecimento com insulina. A mudança de agulha é feita quando o dispositivo é girado em sentido anti-horário, rotacionando o interior e mudando a agulha. A principal desvantagem é a redução do tamanho do reservatório e a troca do dispositivo assim que usadas todas as agulhas, em média 20 dias.

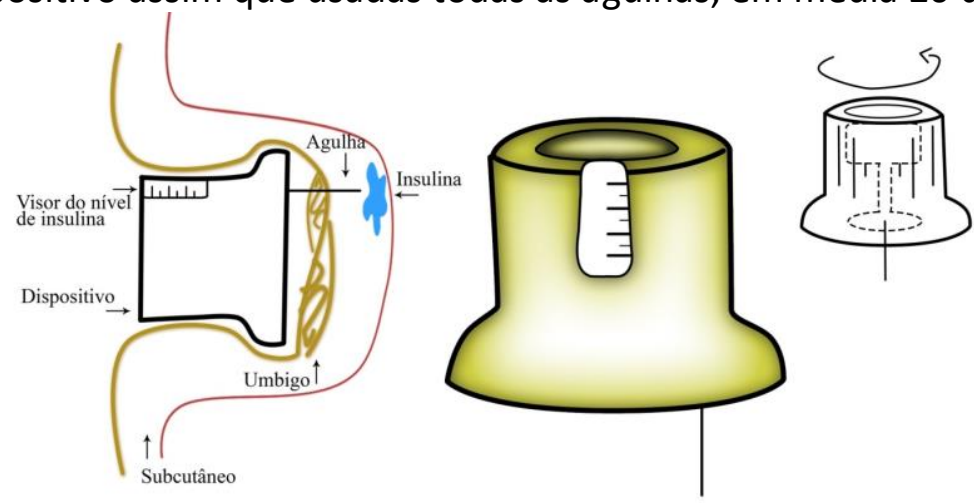

Figura 27 - Conjunto de imagens do conceito $\mathbf{G}$

Conceito $\mathbf{H}$ - Diferentemente dos outros conceitos, este utiliza uma cânula, mesmo sistema das bombas de infusão, Figura 28. A principal vantagem está no aumento do reservatório de insulina e os custos dos insumos/produção comparado aos outros conceitos, que necessitam de um fabrico específico das agulhas e dos suportes. Sua fixação utiliza espuma com memória de forma ou polímero flexível e antes de ser inserido no umbigo é pressionada, reduzido seu tamanho. Dentro do umbigo é retomado o formato original, expandindo e aderindo as paredes do umbigo.
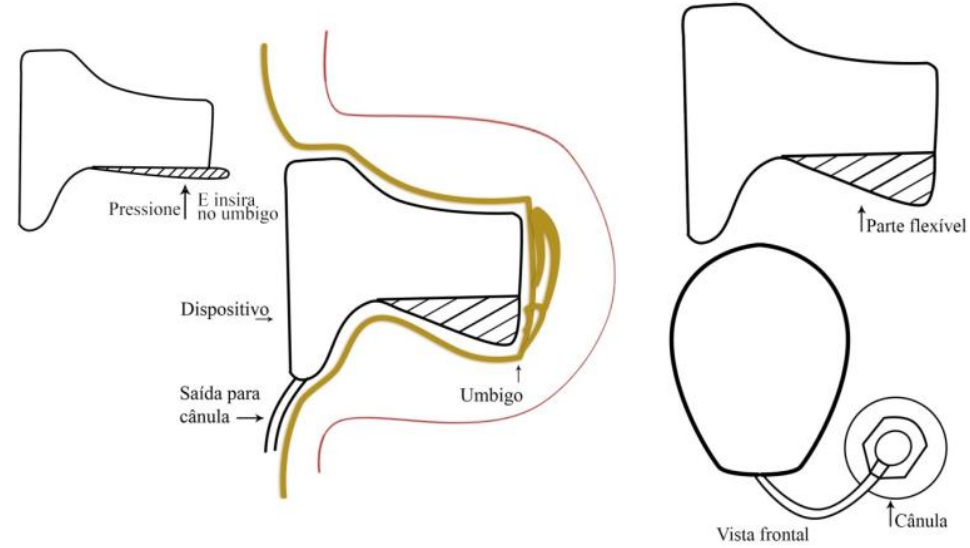

Figura 28 - Conjunto de imagens do conceito H 
Conceito I - Como no conceito anterior, o principal diferencial deste dispositivo é o formato anatômico, não necessitando de outro material para fazer a fixação no umbigo, Figura 29.

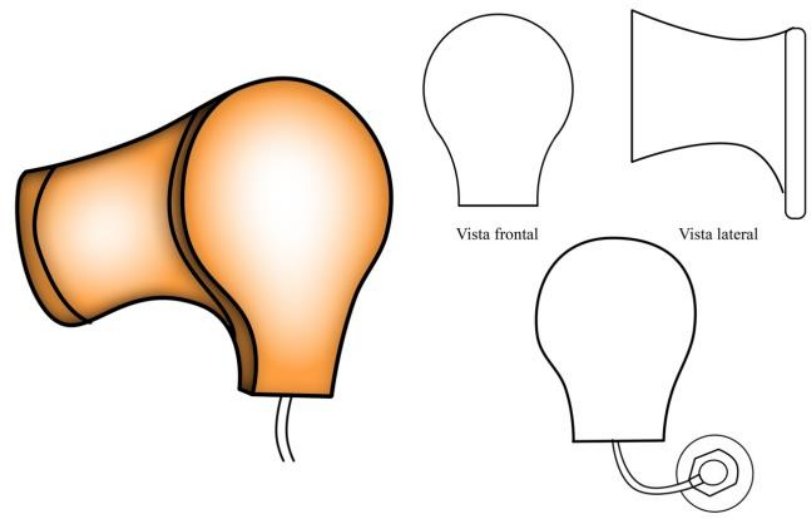

Figura 29 - Conjunto de imagens do conceito I 